\title{
Magnetic helicity of solar active regions
}

\author{
A. Nindos ${ }^{1}$ \\ ${ }^{1}$ Section of Astrogeophysics, Physics Department, University of Ioannina, \\ Ioannina GR-45110, Greece \\ email: anindos@cc.uoi.gr
}

\begin{abstract}
Magnetic helicity is a quantity that describes the linkage and twistedness/shear in the magnetic field. It has the unique feature that it is probably the only physical quantity which is approximately conserved even in resistive MHD. This makes magnetic helicity an ideal tool for the exploration of the physics of eruptive events. The concept of magnetic helicity can be used to monitor the whole history of a CME event from the emergence of twisted magnetic flux from the convective zone to the eruption and propagation of the CME into interplanetary space. In this article, I discuss the sources of the magnetic helicity injected into active regions and the role of magnetic helicity in the initiation of solar eruptions.
\end{abstract}

Keywords. Sun: activity, Sun: magnetic fields, Sun: coronal mass ejections (CMEs), Sun: flares

\section{Introduction}

Magnetic helicity, $H$, quantifies the deviation of a magnetic flux tube from its minimum energy state which corresponds to potential magnetic field. In other words it tells us how much a magnetic flux tube is sheared or/and twisted. For an ensemble of magnetic flux tubes, magnetic helicity can be regarded as a measure of the topological complexity of the field giving information about the linkage and twistedness in the field. The "natural" unit of helicity is the square of magnetic flux $\left(\mathrm{Mx}^{2}\right)$ and therefore the helicity of a twisted flux tube with $N$ turns and magnetic flux equal to unity is simply $N$.

It is well established (e.g. see Berger 1984) that magnetic helicity is very well preserved in plasmas with high magnetic Reynolds numbers, even in the presence of dissipative processes such as magnetic reconnection (more accurately, it is approximately conserved on time scales smaller than the global diffusion time scale; see Berger 1984). This property of helicity has important consequences in the evolution of magnetic fields: a stressed magnetic field cannot relax to a potential field. This behavior may have important implications for the initiation of flares and coronal mass ejections (CMEs).

In this article a short review of magnetic helicity of active regions (ARs) is given. For a more detailed review the interested reader is referred to the article by Démoulin (2007). My article is organized as follows. After defining magnetic helicity and its flux, in section 3 I discuss the sources of magnetic helicity that is injected into ARs. The following two sections address problems that are related to its acurate calculation: the computation of maps of helicity flux density (section 4) and the computation of flows that inject helicity into ARs. In section 6, the role of magnetic helicity in the initiation of eruptive events is briefly outlined. Conclusions are presented in section 7 .

\section{Definitions}

\subsection{Magnetic helicity}

For a magnetic field $\mathbf{B}$ fully contained within a volume $V$ (i.e. at any point of its boundary $S$ the normal component $B_{n}=\mathbf{B} \cdot \hat{\mathbf{n}}$ vanishes), magnetic helicity is defined as 


$$
H=\int_{V} \mathbf{A} \cdot \mathbf{B} \mathrm{d} V
$$

where $\mathbf{A}$ is the magnetic vector potential $(\mathbf{B}=\nabla \times \mathbf{A})$. $H$ is independent of the gauge selection for $\mathbf{A}$ (i.e. independent of the transformation $\mathbf{A} \rightarrow \mathbf{A}+\nabla \Phi$, where $\Phi$ is any single-valued derivable function of space and time).

In the solar atmosphere magnetic flux passes through $S$ (especially in the photosphere) and therefore the above condition is not satisfied. However, Berger and Field (1984) and Finn and Antonsen (1985) have shown that when $B_{n} \neq 0$ on $S$, we can define a gaugeinvariant relative magnetic helicity (hereafter refered to as helicity) of $\mathbf{B}$ with respect to the magnetic helicity of a reference field $\mathbf{B}_{p}$ having the same distribution of normal magnetic flux on the surface $S$ surrounding $V$ :

$$
H=\int_{V} \mathbf{A} \cdot \mathbf{B} \mathrm{d} V-\int_{V} \mathbf{A}_{p} \cdot \mathbf{B}_{p} \mathrm{~d} V
$$

where $\mathbf{A}_{p}$ is the vector potential of $\mathbf{B}_{p}$. The quantity $H$ does not depend on the common extension of $\mathbf{B}$ and $\mathbf{B}_{p}$ outside $V$. Being a potential field it is a convinient choice for $\mathbf{B}_{p}$. If in addition $\nabla \cdot \mathbf{A}_{p}=0$ and $\left(A_{p}\right)_{n}=0$ on $S$ then the term $\int_{V} \mathbf{A}_{p} \cdot \mathbf{B}_{p} \mathrm{~d} V$ vanishes (Berger 1988), so $H$ has the same expression as in the case of the helicity in closed volumes (eq. $2.1)$.

\subsection{Flux of magnetic helicity}

Generally, the amount of helicity within $V$ can change either due to helicity flux crossing $S$ or/and due to dissipation within $V$. Berger (1984) has demonstrated that the helicity dissipation rate is negligible in all processes taking place in the corona, including reconnection and all non-ideal processes. Helicity's dissipation time scale is the global diffusion time scale and consequently it can be regarded as an almost conserved quantity even in resistive MHD.

In the solar atmosphere $V$ is part of the coronal volume, bounded from below by a portion of the photosphere $S_{p}$ and bounded in the corona by $S_{c}\left(S_{c}=S-S_{p}\right)$. No data can presently provide $\mathbf{B}$ on any $S_{c}$ surface. The helicity flux across $S_{c}$ can only be estimated indirectly by the helicity carried away by CMEs, and estimated in interplanetary space from the associated magnetic clouds. All studies compute the helicity injected at the photospheric level through $S_{p}$. Using the gauge $\nabla \cdot \mathbf{A}_{p}=0$, and selecting the boundary condition $\mathbf{A}_{p} \cdot \hat{\mathbf{n}}=0$ for the vector potential of the potential reference field, Berger \& Field (1984) derived the flux of magnetic helicity through a planar surface:

$$
\frac{d H}{d t}=2 \int_{S_{p}}\left[\left(\mathbf{A}_{p} \cdot \mathbf{B}_{t}\right) v_{n}-\left(\mathbf{A}_{p} \cdot \mathbf{v}_{t}\right) B_{n}\right] \mathrm{d} S,
$$

where $B_{t}$ and $B_{n}$ are the tangential and normal components of the photospheric magnetic field and $v_{t}$ and $v_{n}$ the tangential and normal compoments of the photospheric plasma velocity.

\section{Sources of helicity injected into active regions}

The first term of the right-hand side of eq. (2.3) corresponds to the injection of helicity by advection (i.e. emergence of field lines that cross the photosphere) while the second term (also known as shearing term) is the flux of helicity due to motions parallel to $S$. 
Such motions may come either from differential rotation and/or transient photospheric shearing flows.

Differential rotation was the first mechanism that injects helicity into ARs which was studied (DeVore 2000). Even when a single bipole is considered, differential rotation does not provide a monotonous input of magnetic helicity (DeVore 2000). This is because differential rotation rotates both magnetic polarities on themselves and also changes their relative positions, introducing twist and writhe helicity fluxes, respectively. These fluxes always have opposite signs and similar amplitudes, and therefore partially cancel (Démoulin et al. 2002a). Démoulin et al. (2002b) and Green et al. (2002) studied the long-term evolution of the helicity injected by differential rotation into the coronal part of two active regions which were followed from their birth until they decayed. The helicity injection rate from differential rotation was calculated as the sum of the rotation rate of all pairs of elementary fluxes weighted with their magnetic flux. These studies showed that the contribution of differential rotation to the helicity budget of active regions is small.

The total helicity stored into the corona at a given time can be calculated under the force-free field assumption $(\nabla \times \mathbf{B}=\alpha \mathbf{B})$. The best value of $\alpha, \alpha_{\text {best }}$, is determined by comparing the computed field lines with the observed soft X-ray (SXR) or EUV coronal structures. Then the computation of the coronal helicity is relatively straightforward (Berger 1985; see also Georgoulis \& LaBonte 2007).

When high-cadence photospheric magnetograms are available, the horizontal velocity appearing in eq. (2.3) can be computed using the local correlation tracking (LCT) technique (November \& Simon 1988). Several authors have computed the corresponding helicity injection rate (e.g. Chae 2001; Nindos \& Zhang 2002; Moon et al. 2002a,b; Nindos et al. 2003; Chae et al. 2004). Démoulin \& Berger (2003) have pointed out that with magnetograms one follows the photospheric intersection of the magnetic flux tubes but not the evolution of the plasma (generally the two velocities are different). Consequently, from the observed magnetic evolution we obtain the flux tube motion and not the plasma motion parallel to the photosphere. If $\mathbf{v}_{t}$ is the tangential component of the photospheric plasma velocity and $v_{n}$ the velocity perpendicular to the photosphere, the LCT method detects the velocity of the footpoints of the flux tube which is

$$
\mathbf{u}=\mathbf{v}_{t}-\frac{v_{n}}{B_{n}} \mathbf{B}_{t}
$$

The combination of eq. (3.1) and (2.3) shows that the whole helicity flux density can be retrieved within the accuracy of the calculation. Consequently, one may use the quantity $G_{A}=-2 \mathbf{u} \cdot \mathbf{A}_{p} B_{n}$ as a proxy to the whole helicity flux density.

The study of the helicity budget of active regions requires knowledge of the helicity carried away from them. It has been recongized (e.g. Low 1996) that CMEs are the primary agents that remove helicity from active regions. The helicity content of a CME can be estimated by the change of coronal helicity of the source region during the event (e.g. Mandrini et al. 2005). Inside magnetic clouds $H$ is estimated from in situ measurements of the magnetic field vector. This requires a flux rope model whose parameters are determined by a least square fit to the data because only local measurements are available (e.g. Lepping et al. 1990; Daso et al. 2003; 2006). In practice, in studies of the long-term evolution of helicity of active regions that are linked to at least one magnetic cloud at 1 AU one assumes that the helicity carried away by each CME is equal to the helicity content in the magnetic cloud. Nindos et al. (2003) and Lim et al. (2007) were able to partially reconcile the amount of helicity injected into the corona with the helicity carried away by the CMEs in the active regions they studied. However, the uncertainties of these 
studies are significant primarily due to the large uncertainties in the calculation of the helicity transported away by CMEs.

\section{Maps of helicity flux density}

The quantity $G_{A}=-2 \mathbf{u} \cdot \mathbf{A}_{p} B_{n}$ can be used as a proxy to the helicity flux density (see the discussion in section 3 ). This proxy has been used extensively in several studies (references are given in section 3 ). In all these studies $G_{A}$ maps always appear extremely complex both in space and time, with polarities of both signs present at any time. Pariat et al. (2005) showed that $G_{A}$ is not a real helicity flux density and that its properties introduce artificial polarities of both signs (see middle column of Fig. 1). For example, $G_{A}$ is non-zero even in flows that do not inject any magnetic helicity in the field. The spurious signals appear due to the fact that helicity flux densities per unit surface are not physical quantities. Due to the properties of helicity, only helicity flux density per unit of elementary magnetic flux has a physical meaning. But to estimate such quantity using real observations, it is necessary to isolate flux tubes and determine their connectivity, which is actually not possible. Thus any definition of a helicity flux density will only be a proxy of the helicity flux density per unit magnetic flux. Pariat et al. (2005) introduced a new proxy for helicity flux density, $G_{\theta}$, which does not suffer from $G_{A}$ 's problems. $G_{\theta}$
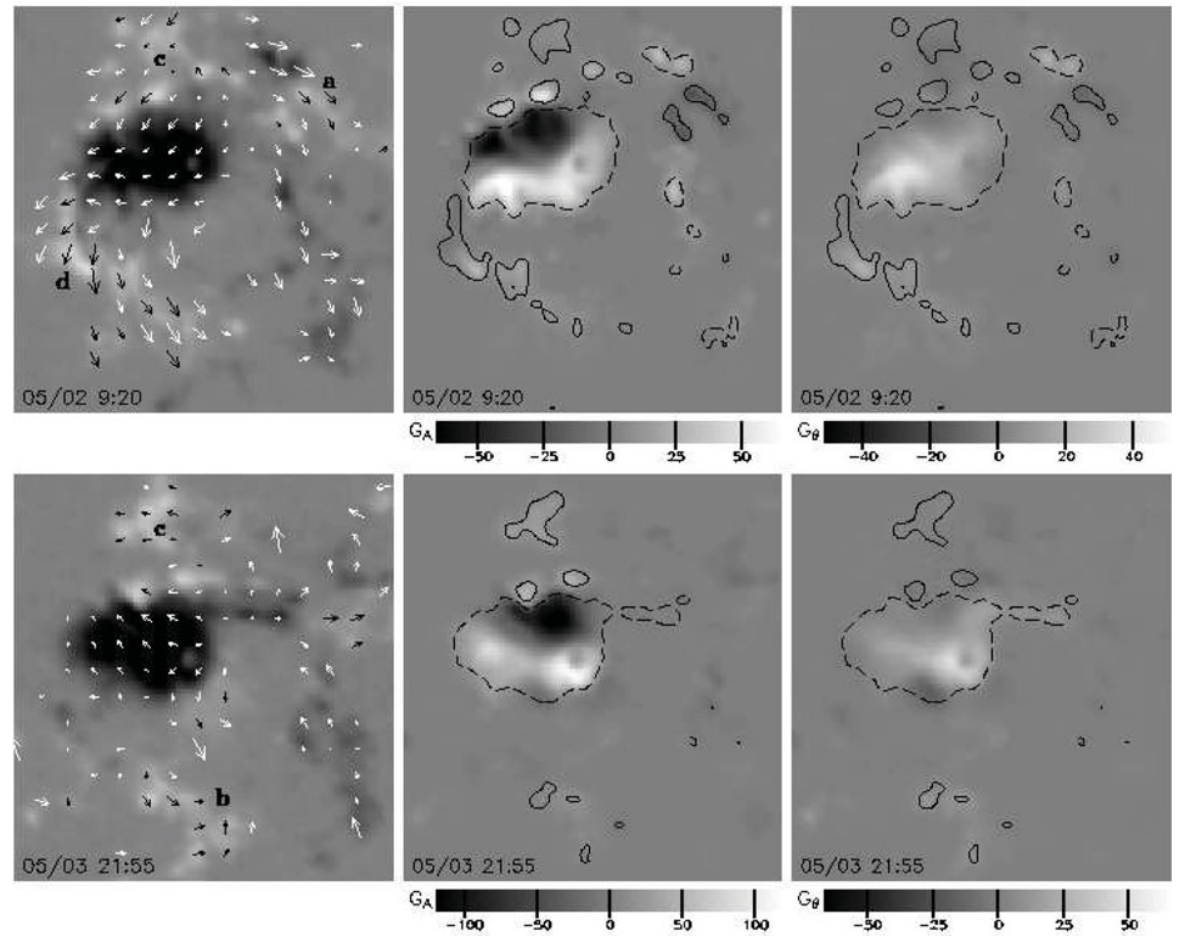

Figure 1. AR 8210 at 09:20 UT on May 2, 1998 (top) and at 21:55 UT on May 3, 1998 (bottom). Left panels: $B_{n}$ magnetograms with velocity field (arrows). Center panels: $G_{A}$ maps. Right panels: $G_{\theta}$ maps. $G_{A}$ and $G_{\theta}$ maps are in units of $10^{6} \mathrm{~Wb}^{2} \mathrm{~m}^{-2} s^{-1}$ and have $\pm 300 \mathrm{G}$ isocontours of $B_{n}$. Note that the scale is not the same for the $G_{A}$ and the $G_{\theta}$ maps (from Pariat et al. 2006). 
implies that the helicity injection rate is the summation of the rotation rate $\frac{\mathrm{d} \theta\left(\mathbf{x}-\mathbf{x}^{\prime}\right)}{\mathrm{d} t}$ of all pairs of elementary fluxes weigthed by their magnetic flux $B_{n} \mathrm{~d}^{2} x$. Therefore it is:

$$
G_{\theta}(\mathbf{x})=-\frac{B_{n}}{2 \pi} \int_{S_{p}} \frac{\mathrm{d} \theta\left(\mathbf{x}-\mathbf{x}^{\prime}\right)}{\mathrm{d} t} B_{n}^{\prime} \mathrm{d}^{2} x^{\prime} .
$$

In order to define the real helicity flux density, the coronal linkage needs to be provided. With it one can represent how all elementary flux tubes move relatively to a given elementary flux tube, and the helicity flux density is defined per elementary flux tube. Using photospheric maps this can be achieved by distributing equally the helicity input between the two footpoints for each elementary flux tube. Then the helicity flux can be rewritten as a flux of magnetic helicity per unit of surface, $G_{\Phi} . G_{\Phi}$ is a field-weighted average of $G_{\theta}$ at both photospheric footpoints, $\mathbf{x}_{ \pm}$, of the photosheric connection:

$$
G_{\Phi}\left(\mathbf{x}_{ \pm}\right)=\frac{1}{2}\left(G_{\theta}\left(\mathbf{x}_{ \pm}\right)+G_{\theta}\left(\mathbf{x}_{\mp}\right)\left|B_{n}\left(\mathbf{x}_{ \pm}\right) / B_{n}\left(\mathbf{x}_{\mp}\right)\right|\right) .
$$

While $G_{\Phi}$ provides the true helicity flux density, its practical use is presently limited by our ability to define the coronal linkage for all magnetic polarities. Currently, all we can do is to estimate $G_{\Phi}$ maps for models that resemble certain configurations and evolution patterns (Pariat et al. 2006; see below).

Pariat et al. $(2006 ; 2007)$ computed $G_{A}$ and $G_{\theta}$ maps at several occasions during the evolution of 5 active regions. Unlike the usual $G_{A}$ maps, most of their $G_{\theta}$ maps showed almost unipolar spatial structures (see Fig. 1) because the nondominant helicity flux densities were significantly suppressed. In a few cases the $G_{\theta}$ maps still contained spurious bipolar signals. With further modelling the computed models of $G_{\Phi}$ were again unipolar. The result of injection of helicity with a coherent sign on the AR scale needs to be checked against statistical studies. If future studies confirm it solar dynamo models will need to explain the formation of twisted flux tubes with either positive or negative helicity but not mixed-sign helicity at the spatial scales resolved by the flow computation methods.

On time scales larger than their transient temporal variations, the time evolution of the total helicity fluxes derived from $G_{A}$ and $G_{\theta}$ show small differences (see Fig. 2 ). Theoretically one expects that the helicity flux integrated using $G_{A}$ and using $G_{\theta}$ should be identical because both definitions are derived from eq. (2.3). The reported small differences may result from the computation of $\mathbf{A}_{p}$ with a fast Fourier transform of the magnetogram which implies an implicit periodicity of the magnetic flux distribution while with the $G_{\theta}$ computation one assumes that no magnetic flux is present around the magnetogram. Chae (2007) and Jeong \& Chae (2007) reported that the integration of $G_{A}$ typically overestimates the helicity injection about 10-30\%. Furthermore, unlike $G_{A}$, with $G_{\theta}$ the time evolution of the total flux is determined primarily by the predominantsigned flux while the nondominant-signed flux is roughly stable and probably mostly due to noise (see Fig. 2).

\section{Computation of photospheric flows}

The discussion in sections 3 and 4 indicates that the computation of photospheric flows is an essential ingredient in any attempt to compute the helicity injected into the coronal part of active regions. The traditionally used LCT method has several limitations that lead to underestimation of the computed helicities (e.g. Démoulin \& Berger 2003; Gibson et al. 2004). Furthermore, if one uses eq. (3.1) one cannot separate the contribution of 
the shearing term from the contribution of the advection term to the helicity injected into the corona. Moreover, a note of caution needs to be added regarding the validity of eq. (3.1). In the photosphere there is a sharp stratification of the plasma and also the photosphere is the interface that separates high to low $\beta$ plasmas. It is a question how a flux tube that is no longer buyoant and has larger radius than the local gravitational scale height will cross this region. Clearly, comparison with MHD simulations are required to check which component(s) of helicity flux will be detected by any method that computes photospheric flows. For this purpose an anelastic MHD simulation was used (Welsch et al. 2007; Ravindra et al. 2008; Schuck 2008) and the comparisons showed that mostly the shearing term of eq. (2.3) can be determined. However, this simulation did not capture essential features of flux emergence physics and therefore the reported comparisons should be treated with caution.

Alternative approaches have been developed which attempt to compute separately both the shearing and advection term using photospheric vector magnetograms. Kusano et al. (2002) proposed a method which uses the vertical component of the induction equation. In fact the velocity of flux tubes cannot be deduced fully from the induction equation and part of the velocity is still computed from the LCT method (Welsch et al. 2004). Georgoulis \& LaBonte (2005) introduced a minimum structure reconstruction technique to infer the velocity field vector. Their analysis simultaneously determines the field-aligned flows and enforces a unique cross-field solution of the induction equation.

Longcope (2004) introduced a technique (Minimum Energy Fit method; MEF) which demands that the photospheric flow agree with the observed photospheric field evolution according to the induction equation. It selects from all consistent flows, that with the smallest overall flow speed by demanding that it minimize an energy functional. If partial

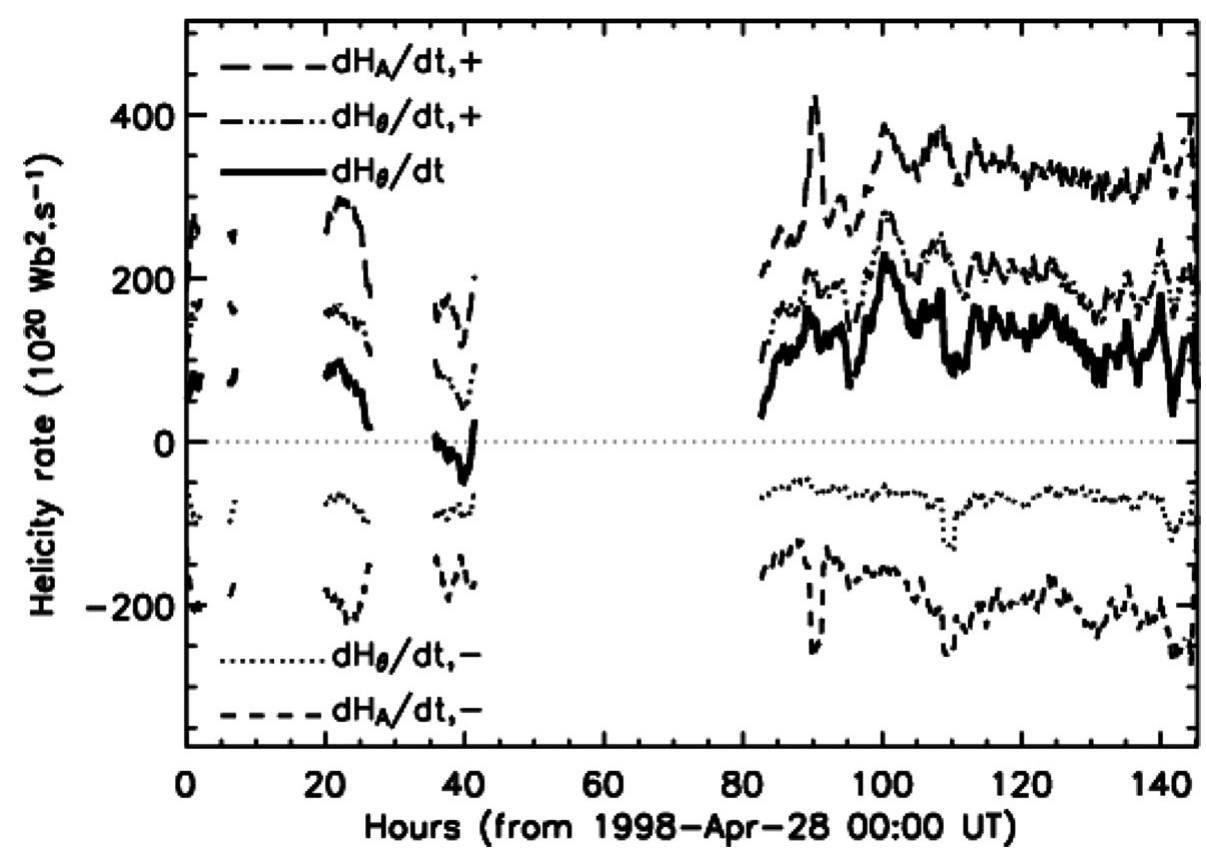

Figure 2. Plots of $\left(d H_{A} / d t\right)_{ \pm},\left(d H_{\theta} / d t\right)_{ \pm}$, and $d H_{\theta} / d t$ as a function of time for AR 8210 . The curves have been smoothed on a time interval of $100 \mathrm{~min}$. We do not present the $d H_{A} / d t$ curve because its differences with respect to the $d H_{\theta} / d t$ curve are too small to be clearly seen (from Pariat et al. 2006). 
velocity information is available from other measurements, it can be incorporated into the MEF methodology by minimizing the squared difference from that data. Ravindra et al. (2008) incorporated velocity information provided by the LCT technique and Doppler velocity measurements. They compared their results with the results of an anelastic MHD simulation (see Fig. 3). The figure shows that LCT largely underestimates the amount of helicity rate while the best performance comes from the MEF method with additional LCT input.

Schuck (2006) developed the Differential Affine Velocity Estimator (DAVE), a method that locally minimizes the square of the continuity equation for the vertical component of the magnetic field subject to an affine velocity profile. Schuck (2008) presented the extension of the DAVE for horizontal magnetic fields, with all plasma components $\mathbf{v}_{t}$ and $v_{n}$ described by a local model with linear spatial variations. The new method is called DAVE4VM (DAVE for vector magnetograms) because it requires input from vector magnetogram data.

The above methods, except DAVE4VM, were checked against an anelastic MHD simulation (Welsch et al. 2007). The method with the best overall performance was the MEF method. All methods showed weak features that have pointed out by Welsch et al. (2007). Schuck (2008) checked the DAVE4VM method against the same anelastic MHD simulation used by Welsch et al. (2007) and found that his method could reproduce roughly $95 \%$ of the simulation's helicity rates.

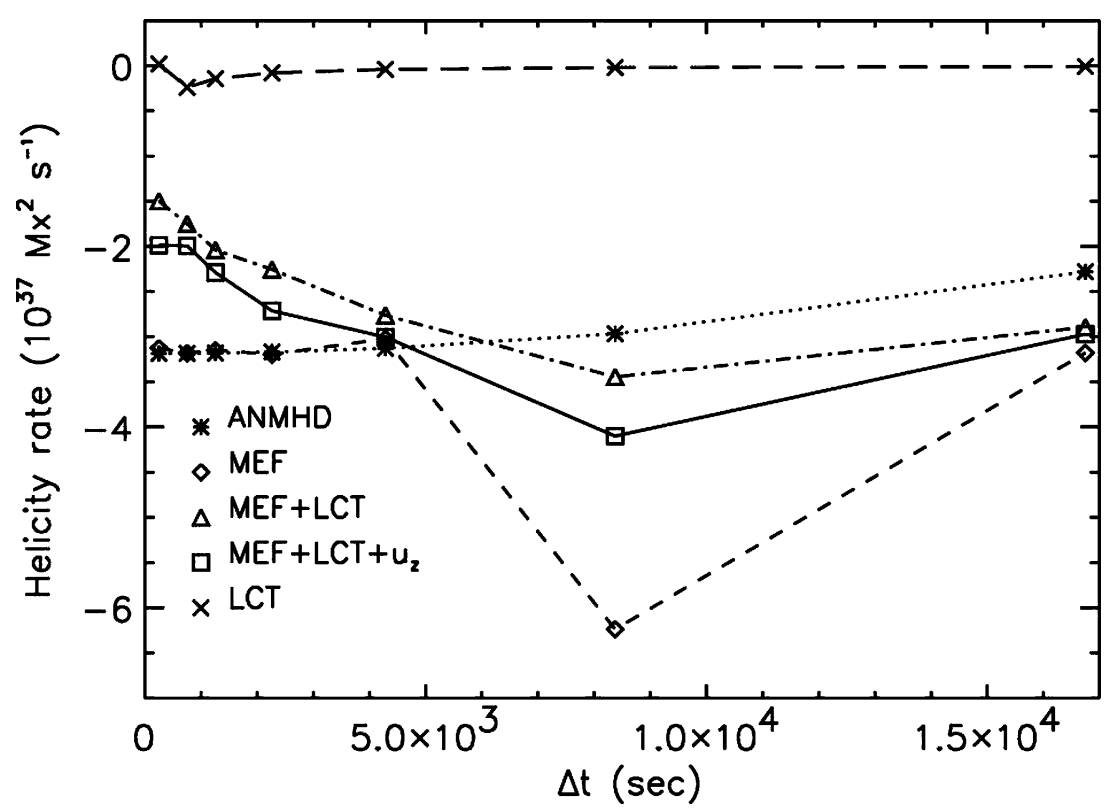

Figure 3. Helicity fluxes obtained by using LCT velocity and various combinations of the MEF algorithm with all velocities plotted as a function of time. The true helicity fluxes (labeled ANMHD) that resulted from an anelastic MHD simulation are also plotted for comparison. The curves labeled MEF, MEF $+\mathrm{LCT}, \mathrm{MEF}+\mathrm{LCT}+u_{z}$ indicate helicity fluxes computed with MEF without additional data, MEF with additional LCT method data, and MEF with additional LCT method data and additional data for the vertical component of the velocity field provided by the anelastic MHD simulation (from Ravindra et al. 2008). 


\section{Magnetic helicity and CME initiation}

A significant fraction of AR's helicity is created by the solar dynamo and then transported into the corona through the photosphere with the emerging magnetic flux. This process together with helicity's property not to be destroyed under reconnection would constantly accumulate helicity into the corona. Furthermore, on the global scale, helicity emerges predominantly negative in the northern hemisphere and predominantly positive in the southern hemisphere (e.g. Pevtsov et al. 1995). And also this hemispheric helicity sign pattern does not change from solar cycle to solar cycle (Pevtsov et al. 2001). Consequently, on the global scale, mutual cancellation of helicity of opposite signs cannot relieve the Sun from excess accumulated helicity. It has been suggested (e.g. Low 1996) that CMEs, as expulsions of twisted magnetic fields, consist the most important process through which accumulated helicity is removed from the corona. Indirect support for this scenario is provided by the work by Zhang et al. (2006) who concluded that there is always a maximum amount of helicity that can be stored in an axisymmetric force-free field outside a sphere.

Low \& Zhang (2002) and Zhang \& Low (2001; 2003) provided a unified view of CMEs as the last chain of processes that transfer helicity from the convective zone into the interplanetary medium. Their theory exploits Taylor's conjecture that the magnetic field will relax towards a linear force-free field state. A summary of their results is as follows. When new field enters the corona repeated reconnections between the new and preexisting field take place. This process simplifies the magnetic topology and the dissipated magnetic energy produces flares. The relaxation proceeds according to Taylor's conjecture and results in the formation of a flux rope which contains a significant fraction of the total helicity of the system. The fate of the flux rope is determined by the efficiency of its confinement by its surrounding anchored field. Flux rope ejection occurs when
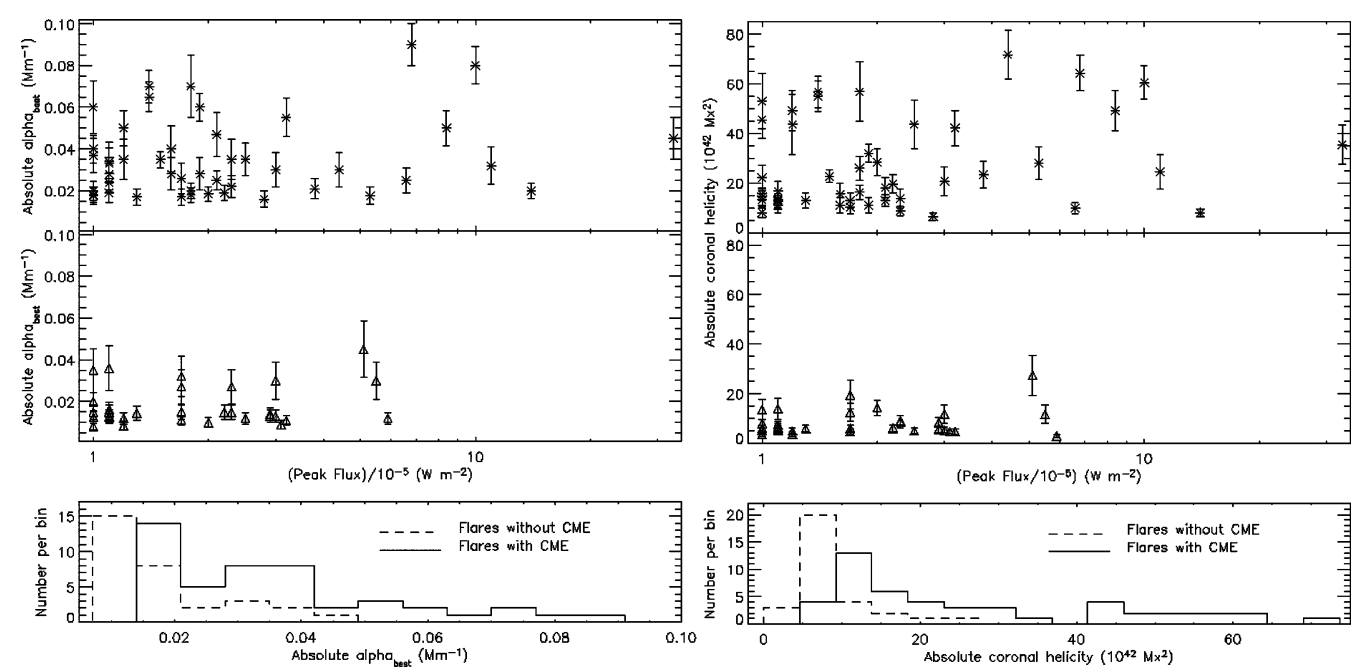

Figure 4. Left column, top: Scatter plot of the pre-flare absolute values of $\alpha_{b e s t}$ as a function of the flare's peak X-ray flux for ARs producing CME-associated flares. Left column, middle: Same as top panel, but for the ARs producing flares without CMEs. Left column, bottom: Histograms of the values of $\alpha_{b e s t}$ appearing in the top and middle panels. The solid line represents the histogram of $\alpha_{b e s t}$ of the ARs which give CME-associated flares while the dashed line is the histogram of $\alpha_{b e s t}$ of the ARs which produce flares that do not have CMEs. Right column: The absolute coronal helicity of the ARs appearing in the left panel. The format is identical to the format of the left column (modified from Nindos \& Andrews 2004). 
the magnetic energy it contains is sufficient to drive an outward expansion against the confining field.

The above physical view is supported by the work by Nindos \& Andrews (2004). They modeled under the linear force-free field approximation the pre-flare coronal field of 78 ARs that produced big flares. Only some $60 \%$ of these flares were associated with CMEs. Then from the derived values of $\alpha_{\text {best }}$ they computed the corresponding coronal helicities. Their results appear in Fig. 4 and indicate that in a statistical sense both the pre-flare absolute value of $\alpha$ and the corresponding coronal helicity of the ARs producing CMEassociated big flares are larger than the absolute value of $\alpha$ and helicity of those that do not have associated CMEs.

There are several other approaches to the initation of CMEs and the role played by magnetic helicity. Amari et al. (2003a,b) concluded that the accumulation of helicity is a necessary but not sufficient condition for an eruption to occur. The breakout simulations by Phillips et al. (2005) were designed so that no global helicity was injected into the corona. They showed that the eruption occurs at almost the same magnetic energy threshold as in a previous simulation where only positive helicity was injected. In their simulation, the amount of helicity is irrelevant because the negative and positive helicity regions did not reconnect. Contrasting results were found in the simulation by Kusano et al. (2004) where the introduction of a reverse helicity is essential for the eruption of a sheared arcade.

\section{Conclusions}

Magnetic helicity has the unique feature of being conserved even in resistive MHD on time scales less than the global diffusion time scale. This makes helicity probably the only physical quantity which can monitor the entire history of an eruptive event: from the transfer of magnetic field from the convective zone all the way to the eruption and the escape of the CME into interplanetary medium. On the other hand, calculations of helicity are difficult and only relatively recently attempts have been made to measure helicity using solar observations.

Once the importance of helicity was realized, a lot of effort was put on the determination of the sources of the helicity injected into active regions. Theoretical considerations have demonstrated rigorously that shearing motions (either differential rotation or/and transient flows) on the photospheric surface is an inefficient way of providing helicity on the active region scale. However, computations using high-cadence longitudinal magnetograms give the total helicity flux and cannot separate the shearing from the advection term. Furthermore, the computation of velocitites using the LCT method has serious limitations. Attempts for the computation of the shearing and advection term separately have been made using vector magnetograms. But the algorithms that have been developed have not been applied extensively to observations. Even more serious uncertainties are associated with the computation of the helicity carried away by CMEs. All the above problems contribute to the discrepancies concerning the helicity budget of ARs. At this point, these uncertainties have been cleared up only partially and much work needs to be done on this issue.

The role of helicity in the initiation of solar eruptions is a theoretical subject of intense debate. There is a general consensus that for the CME initiation, helicity must be accumulated into the pre-eruption topology. However, it seems that other parameters are also important, for example the location with respect to the pre-existing field where helicity is injected, the efficiency of the reconnection process(es) and how efficiently the helicity-charged stucture is confined by the overlying magnetic field. 


\section{References}

Amari, T., Luciani, J. F., Aly, J. J., Mikic, Z., \& Linker, J. 2003a, ApJ, 585, 1073

Amari, T., Luciani, J. F., Aly, J. J., Mikic, Z., \& Linker, J. 2003b, ApJ, 595, 1231

Berger, M. A. 1984 Geophys. Astrophys. Fluid Dyn., 30, 79

Berger, M. A. 1985 ApJS, m59, 433

Berger, M. A. $1988 A \mathscr{G} A, 201,355$

Berger, M. A., \& Field, G. B. 1984 J. Fluid Mech., 147, 133

Chae, J. 2001 ApJ (Letters), 560, L95

Chae, J. 2007 Adv. Sp. Res., 39, 1700

Chae, J., Moon, Y.-J., \& Park, Y.-D. 2004 Solar Phys., 223, 39

Dasso, S., Mandrini, C. H., Démoulin, P., \& Farrugia, C. J. 2003 JGR, 108, 1362

Dasso, S., Mandrini, C. H., Démoulin, P., \& Luoni, M. L. 2006 A\&A, 455, 349

Démoulin, P. 2007 Adv. Sp. Res., 39, 1674

Démoulin, P. \& Berger, M. A. 2003 Solar Phys. 215, 203

Démoulin, P., Mandrini, C. H., van Driel-Gesztelyi, L., Lopez-Fuentes, M. C, \& Aulanier, G. 2002a Solar Phys. 207, 87

Démoulin, P., Mandrini, C. H., van Driel-Gesztelyi, L., et al. 2002b A\&\&A, 382, 650

DeVore, R. C. 2000 ApJ, 539, 944

Finn, J. H. \& Antonsen, T. M. J. 1985 Comments Plasma Phys. Contr. Fus., 9, 111

Georgoulis, M. K. \& LaBonte, B. J. 2006 ApJ, 636, 475

Georgoulis, M. K. \& LaBonte, B. J. 2007 ApJ, 671, 1034

Gibson, S. E., Fan, Y., Mandrini, C. H., Fisher, G., \& Démoulin, P. 2004 ApJ, 617, 600

Green, L. M., Lopez-Fuentes, M. C., Mandrini, C. H., et al. 2002 Solar Phys., 208, 43

Jeong, H. \& Chae, J. 2007 ApJ, 671, 1022

Kusano, K., Maeshiro, T., Yokoyama, T., \& Sakurai, T. 2002 ApJ, 577, 501

Kusano, K., Maeshiro, T., Yokoyama, T., \& Sakurai, T. 2004 ApJ, 610, 537

Lepping, R. P., Burlaga, L. F., \& Jones, J. A. 1990 JGR, 95, 11957

Lim, E.-K., Jeong, H., Chae, J., \& Moon, Y.-J. 2007 ApJ, 656, 1167

Longcope, D. W. 2004 ApJ, 612, 1181

Low, B. C. 1996 Solar Phys., 167, 217

Low, B. C. \& Zhang, M. 2002 ApJ (Letters), 564, L53

Mandrini, C. H., Pohjolainen, S., Dasso, S., et al. 2005 A $\& A$, 434, 725

Moon, Y.-J., Chae, J., Choe, G. S., et al. 2002a ApJ, 574, 1066

Moon, Y.-J., Chae, J., Wang, H., Choe, G. S., \& Park, Y. D. 2002b ApJ, 580, 528

Nindos, A. \& Zhang, H. 2002 ApJ (Letters), 573, L133

Nindos, A., Zhang, J., \& Zhang, H. 2003 ApJ, 594, 1033

Nindos, A. \& Andrews, M. D. $2004 A p J$ (Letters), 616, L175

November, L. J. \& Simon, G. W. 1988 ApJ, 333, 427

Pariat, E., Démoulin, P., \& Berger, M. A. 2005 A $6 A, 439,1191$

Pariat, E., Démoulin, P., \& Nindos, A. 2007 Adv. Sp. Res., 39, 1706

Pariat, E., Nindos, A., Démoulin, P., \& Berger, M.A. 2006 A 6 A, 452, 623

Pevtsov, A. A., Canfield, R. C., \& Metcalf, T. R. 1995 ApJ (Letters), 440, L109

Pevtsov, A. A., Canfield, R. C., \& Latushko, S. M. 2001 ApJ (Letters), 549, L261

Phillips, A. D., MacNeice, P. J., \& Antiochos, S. K. 2005 ApJ (Letters), 624, L129

Schuck, P. W. 2006 ApJ, 646, 1358

Schuck, P. W. 2008 ApJ, 683, 1134

Ravindra, B., Longcope, D. W., \& Abbett, W. P. 2008 ApJ, 677, 751

Welsch, B. T., Abbett, W. P., DeRosa, M. L., et al. 2007 ApJ, 670, 1434

Welsch, B. T., Fisher, G. H., Abbett, W. P., \& Regnier, S. 2004 ApJ, 610, 1148

Zhang, M. \& Low, B. C. 2001 ApJ, 561, 406

Zhang, M. \& Low, B. C. 2003 ApJ, 584, 479

Zhang, M., Flyer, N., \& Low, B. C. 2006 ApJ, 644, 575 


\section{Discussion}

DAVILA: Pevtsov et al. (2003) report a good temporal correlation between flux emergence and helicity increase in active regions, indicating a subsurface origin of helicity. What is the basis of the doubts you expressed in this regard?

Nindos: There is no doubt, theoretically, that flux emergence is the most efficient agent. But what do our techniques really measure? In this question, Pevtsov's work simply handles mean parameters.

GIRISH: I want to know whether your model of helicity and magnetic flux carried by CMEs into the corona is valid for high latitude CMEs observed during sunspot maximum outside the active region belt on the Sun?

Nindos: Yes it is, because "ARs" even when they contain no spots or plages still have helicity. 